\title{
Fish odour syndrome
}

\author{
Min Li MD PhD, Ahmad Al-Sarraf MD, Graham Sinclair PhD, Jiri Frohlich MD
}

A 68-year-old woman with familial hypercholesterolemia and osteoarthritis was treated with atorvastatin $10 \mathrm{mg}$ daily for four months, ending in October 2006. At that time, hypothyroidism was diagnosed, and the atorvastatin was temporarily discontinued. She returned to our lipid clinic in September 2009, because her total and low-density-lipoprotein [LDL] cholesterol levels were significantly increased. At this point, rosuvastatin $10 \mathrm{mg}$ daily was started.

Two weeks after starting the rosuvastatin, the patient noticed a strong fish odour, similar to a problem she had experienced before menopause. When she was nine years old, she had undergone investigation for a strong odour of rotting fish, for which she was ostracized by her classmates. The smell had decreased after puberty, but she still noticed a strong fishy smell around the time of her menstrual periods and when she was eating a choline-rich diet, such as fish and eggs. The smell had disappeared after menopause.

At the time when the smell returned, shortly after initiation of rosuvastatin, the patient had been receiving levothyroxine therapy for about three years (since October 2006), and there had been no change in her diet. After two months of rosuvastatin therapy, the smell became intolerable and she stopped taking the drug. Three days later the smell disappeared. Laboratory testing showed that the patient's liver and kidney functions were all within normal ranges. As would be expected with treatment with a lipid-lowering agent, there had been a decrease in her LDL cholesterol (from $6.9 \mathrm{mmol} / \mathrm{L}$ at baseline to 3.2 $\mathrm{mmol} / \mathrm{L}$, i.e., a $53.6 \%$ reduction).

Primary trimethylaminuria (fish odour syndrome) was suspected. Analysis of a urine sample obtained after the patient had stopped taking rosuvastatin revealed elevated excretion of trimethylamine and a lower-than-normal percent ratio of trimethylamine $\mathrm{N}$-oxide to trimethylamine (92.4:7.6 [normally > 97:3]), as measured by fast atom bombardment mass spectrometry. ${ }^{1}$ Subsequent molecular analysis of the coding region of the FMO3 gene (for flavin-containing monooxygenase 3 ) revealed compound hetero- zygosity for one known pathogenic mutation (p.P153L, c.458C > T) and one novel substitution (p.I8T, c.23T $>$ C). The p.I8T substitution is not a known polymorphism for trimethylaminuria, but it may be pathogenic. The patient was also heterozygous for a common variant haplotype (p.[E158K;E308G]) that has been shown to exacerbate excretion of trimethylamine in association with other disease-causing mutations. ${ }^{2}$ Pravastatin $20 \mathrm{mg}$ daily was started, and no symptoms were reported after 16 weeks of treatment.

\section{Discussion}

Common causes of a fishy odour include poor hygiene, gingivitis, bacterial vaginosis and urinary tract infections. Advanced kidney or liver disease may also cause this condition. ${ }^{3}$ In the patient described here, a less common cause was found. Trimethylaminuria, also known as fish odour syndrome, is a metabolic disorder first described in $1970 .{ }^{4}$ It is characterized by abnormal excretion of trimethylamine in the urine, breath, sweat and vaginal secretions. It may be primary in origin or secondary to liver or kidney damage, or it may be caused by an increase in the precursors of trimethylamine, such as choline, lecithin or carnitine in the diet. ${ }^{4}$

The primary syndrome is inherited in an autosomal recessive manner. The defective enzyme is flavin-containing monooxygenase 3 , the gene for which $(F M O 3)$ is located in chromosome region 1q23-25. ${ }^{5}$ Trimethylamine is derived from the intestinal bacterial degradation of foods rich in choline, lecithin and carnitine. Trimethyl-

\section{KEY POINTS}

- Primary trimethylaminuria (fish odour syndrome) is a metabolic disorder that can be exacerbated by menstruation, specific medications or the intake of choline-rich foods.

- More common causes of a fishy odour include poor hygiene, gingivitis, vaginosis, urinary tract infections, and advanced liver and kidney disease.

- Management of trimethylaminuria includes dietary modifications, use of mildly acidic soaps, avoidance of certain medications and possibly a short course of neomycin or metronidazole. 
amine produced within the intestinal tract is rapidly absorbed and is converted within the liver, by the action of flavin-containing monooxygenase 3 , to trimethylamine $N$-oxide (Figure 1), which is then excreted in the urine. Affected individuals have a reduced capacity to metabolize trimethylamine into trimethylamine $\mathrm{N}$-oxide. Excessive amounts of the volatile molecule are therefore excreted in the body fluids, which give off a strong fishy smell.

Trimethylaminuria was previously considered to be rare, but evidence suggests that its prevalence is much higher than initially thought. The incidence of heterozygous carriers of the allele for trimethylaminuria has been studied in relatively few populations. The incidence of heterozygous carriers in the white British population has been estimated to be as high as $1 \%,{ }^{5}$ and the carrier rate may be as high as $3 \%$ in Ecuador and $11 \%$ in New Guinea. ${ }^{5}$ Carriers may have transient episodes or mild symptoms of fish odour syndrome. $^{4}$

\section{Clues to the diagnosis}

The rotten fish odour is usually present from childhood and is exacerbated during puberty. In women, the offensive odour may be enhanced by oral contraceptives or may increase just before and during menstruation as a result of hormonal inhibition of the oxidation of trimethylamine. Patients with trimethylaminuria have no physical

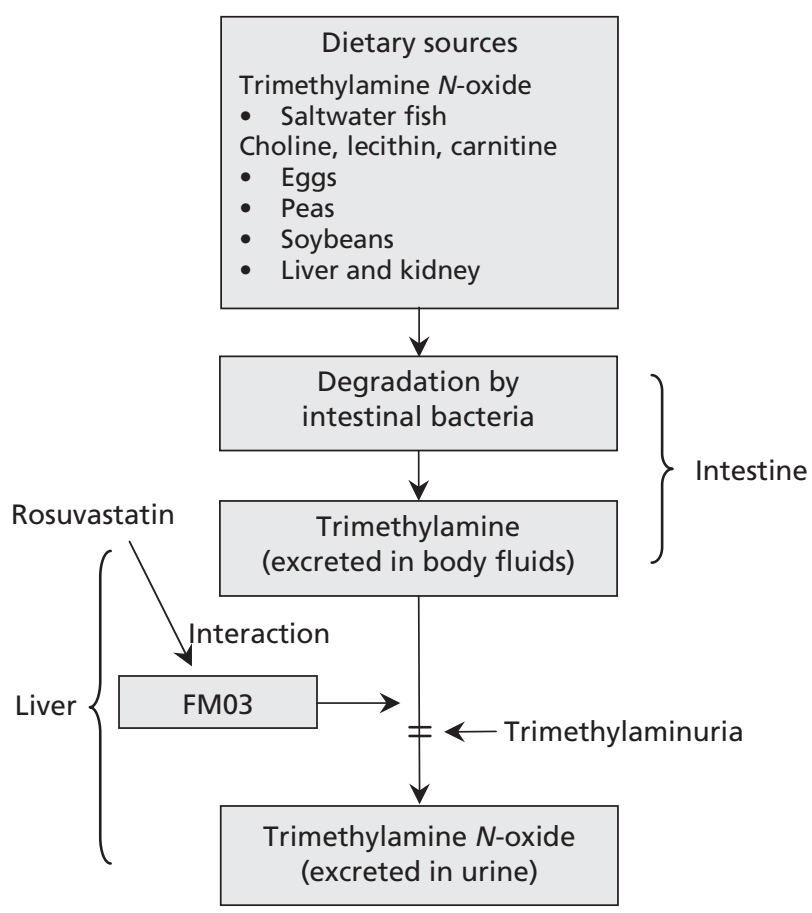

Figure 1: Schematic presentation of the metabolic pathway of trimethylamine. FMO3 = flavin-containing monooxygenase 3 . abnormality. However, the unpleasant odour can result in a variety of psychosocial problems, including withdrawn personality, social isolation, obsessive personal cleansing, disruption of schooling, marital disharmony, clinical depression and suicidal tendencies. ${ }^{6}$

As mentioned previously, trimethylaminuria should be differentiated from more common causes of a fishy odour, such as poor hygiene, gingivitis, vaginosis, urinary tract infections, and advanced liver and kidney disease. ${ }^{3}$

The biochemical diagnosis is established by measuring the ratio of trimethylamine $N$-oxide to trimethylamine in the urine. Among people without trimethylaminuria, more than $97 \%$ of excretion occurs as trimethylamine $\mathrm{N}$-oxide. In patients with the condition, the ratio is reduced. To date, several different mutations of the FMO3 gene have been reported to cause fish odour syndrome. ${ }^{5}$

\section{Treatment options}

Treatment includes counselling and dietary modifications. An explanation of the biochemical nature of the disorder and the exacerbating factors such as menstruation may assist in relieving patients' anxiety. Behavioural counselling may help with depression and other psychological symptoms. Genetic counselling should be considered if the patient has the primary form of the syndrome. Dietary adjustments include avoidance of choline-rich foods, such as egg yolk, liver, kidney, peas, soybeans and sea fish. ${ }^{3}$

Expert opinion suggests that a short course of low-dose neomycin or metronidazole can be used to suppress production of trimethylamine in the gut. ${ }^{7}$ The use of mildly acidic soaps may help to reduce the odour in some patients. ${ }^{8}$ Additional therapeutic strategies that may be developed in the future include gene therapy, attempts to colonize the human gut with microorganisms engineered with human flavin-containing monooxygenase 3 and enzyme induction with drugs. ${ }^{9}$

\section{Exacerbation with rosuvastatin}

Flavin-containing monooxygenase 3 is known to be involved in the nicotinamide adenine dinucleotide phosphate-dependent oxidation and metabolism of a number of drugs such as tamoxifen, ketoconazole, sulindac sulphide and benzydamine. ${ }^{10}$ Given this involvement, case reports of exacerbation of fish odour syndrome in association with these drugs and other medications might be expected, because patients with primary trimethylaminuria may have a reduced ability to metabolize these compounds.

In the patient described here, the symptoms were exacerbated by rosuvastatin. Only $10 \%$ of rosuvastatin is metabolized in the liver, and the 
remainder is excreted unchanged. ${ }^{11}$ At the moment, we do not know the mechanism or mechanisms by which rosuvastatin exacerbates the symptoms of fish odour syndrome. Rosuvastatin contains a tertiary amine, which is similar in structure to trimethylamine, whereas none of the other statins, such as atorvastatin and pravastatin, have a tertiary amine structure. We hypothesize that rosuvastatin or one of its metabolites interacts with flavin-containing monooxygenase 3 , competitively inhibiting the enzyme activity and exacerbating the fishy smell. Rosuvastatin may also exacerbate the symptoms of trimethylaminuria through a competitive transport process. It has been shown that drugs that interact with P-glycoprotein (such as rosuvastatin) inhibit the intestinal import of choline. ${ }^{12}$ This impaired import of choline from the intestine could result in increased bacterial production of trimethylamine in the intestine.

\section{References}

1. Mamer OA, Chioniere L, Treacy EP. Measurement of trimethylamine and trimethylamine $N$-oxide independently in urine by fast atom bombardment mass spectrometry. Anal Biochem 1999; 276:144-9.

2. Zhou J, Shephard EA. Mutation, polymorphism and perspec- tives for the future of human flavin-containing monooxygenase 3. Mutat Res 2006;612:165-71

3. Rehman HU. Fish odor syndrome. Postgrad Med J 1999;75:451-2

4. Mitchell SC, Smith RL. Trimethylaminuria: the fish malodor syndrome. Drug Metab Dispos 2001;29:517-21.

5. Hernandez D, Addou S, Lee D, et al. Trimethylaminuria and a human FMO3 mutation database. Hum Mutat 2003;22:209-13.

6. Todd WA. Psychosocial problems as the major complication of an adolescent with trimethylaminuria. J Pediatr 1979;94:497-501.

7. Ayesh R, Mitchell SC, Zhang AQ, et al. The fish odour syndrome: biochemical, familial and clinical aspects. BMJ 1993;307:655-7.

8. Wilcken B. Acid soaps in the fish odour syndrome. BMJ 1993; 307:1497.

9. Arseculeratne G, Wong AK, Goudie DR, et al. Trimethylaminuria (fish-odor syndrome): a case report. Arch Dermatol 2007;143:81-4.

10. Cashman JR, Zhang J. Interindividual differences of human flavin-containing monooxygenase 3 : genetic polymorphisms and functional variation. Drug Metab Dispos 2002;30:1043-52.

11. Martin PD, Warwick MJ, Dane AL, et al. Metabolism, excretion, and pharmacokinetics of rosuvastatin in healthy adult male volunteers. Clin Ther 2003;25:2822-35.

12. Kamath AV, Darling IM, Morris ME. Choline uptake in human intestinal Caco-2 cells is carrier-mediated. J Nutr 2003;133: 2607-11.

Affiliations: From the Healthy Heart Program Prevention Clinic (Li, Al-Sarraf, Frohlich), St. Paul's Hospital, Vancouver, BC; the Department of Pathology and Laboratory Medicine (Al-Sarraf, Frohlich), Faculty of Medicine, University of British Columbia; and the Department of Pathology (Sinclair), BC Children's Hospital, Vancouver, BC

Contributors: All of the authors participated equally in writing the article and revising it critically for important intellectual content, and all approved the final version submitted for publication. 